\title{
Light deflection in the second post-Newtonian approximation of scalar-tensor theory of gravity
}

\author{
P. Dong ${ }^{1,2}$ and W. T. Ni ${ }^{1}$ \\ ${ }^{1}$ Center for Gravitation and Cosmology, Purple Mountain Observatory, \\ Chinese Academy of Sciences, Nanjing, 210008, China \\ emails: dongpeng@pmo.ac.cn, wtni@pmo.ac.cn \\ ${ }^{2}$ Graduate University of the Chinese Academy of Sciences, Beijing, 100049, China
}

\begin{abstract}
In this paper, we use the metric coefficients and the equation of motion in the 2nd post-Newtonian approximation in scalar-tensor theory including intermediate range gravity to derive the deflection of light and compare it with previous works. These results will be useful for precision astrometry missions like Gaia, SIM, and LATOR (Laser Astrometric Test Of Relativity) which aim at astrometry with micro-arcsecond and nano-arcsecond accuracies and a need for the 2nd post-Newtonian framework and ephemeris to determine the stellar and spacecraft positions.
\end{abstract}

Keywords. astrometry, reference systems, time

\section{Introduction}

The relativistic light deflection passing near the solar rim is 1.75 as (arcsec). The first post-Newtonian approximation is valid to $10^{-6}$ and the second post-Newtonian is valid to $10^{-12}$ of relativistic effects such as light deflection in the solar system. For astrometry mission to measure angles with an accuracy in the range of nano-as to $\mu$ as, the 2 nd post-Newtonian approximation of relevant theories of gravity is required for both the angular measurement and relativistic gravity tests. The scalar-tensor theory is widely discussed and used in tests of relativistic gravity. In order to confront the predictions of scalar-tensor theory with experiment in the solar system, it is necessary to compute its second post-Newtonian approximation and certain gravitational effects such as deflection of light, time delay of light and perihelion shift. The 2nd post-Newtonian contribution for light ray has been discussed by and Epstein \& Shapiro (1980), Richter \& Matzner (1982) and by others. In this paper, we use the metric coefficients we obtained earlier (Xie et al. 2007) to compute the deflection in the second post-Newtonian approximation considering the velocity of the observer (spacecraft).

\section{Metric coefficients}

The calculation of light deflection to $2 \mathrm{PN}$ approximation requires knowledge of terms in the metric to order $(v / c)^{4}$. For the scalar-tensor theory, the metric coefficients are

$$
g_{00}=1-2 U+2(1+\bar{\beta}) U^{2}, g_{i j}=-\delta_{i j}\left[1+2(1+\bar{\gamma}) U+\frac{3}{2}(1+\Lambda) U^{2}\right]
$$

in the global coordinates for the static case. Note that $U$ is given by

$$
U=\int \frac{\rho\left(\vec{x}^{\prime}, t\right)}{\left|\vec{x}-\vec{x}^{\prime}\right|} d^{3} x^{\prime}-\frac{\bar{\gamma} \xi_{1}}{4 \pi} \int \frac{d^{3} x^{\prime}}{\left|\vec{x}-\vec{x}^{\prime}\right|} \int \frac{\rho\left(\vec{x}^{\prime \prime}, t\right)}{\left|\vec{x}^{\prime}-\vec{x}^{\prime \prime}\right|} \exp \left[-\xi_{1}\left(\vec{x}^{\prime}-\vec{x}^{\prime \prime}\right)\right] d^{3} x^{\prime \prime}
$$


and the parameters $\bar{\gamma}, \bar{\beta}, \xi_{1}$ and $\Lambda$ are given in Xie et al. (2007) by

$$
\bar{\gamma}=-\frac{1}{\omega_{0}+2}, \bar{\beta}=\frac{\omega_{1}}{\left(2 \omega_{0}+3\right)\left(2 \omega_{0}+4\right)^{2}}, \Lambda=\frac{15}{6} \bar{\gamma}+\frac{4}{3}\left(\bar{\gamma}^{2}+\bar{\beta}\right), \xi_{1}=-4 \frac{\bar{\gamma} \lambda_{2} \phi_{0}^{2}}{2+\bar{\gamma}}
$$

\section{Deflection angle}

The basic equations of a light ray trajectory are

$$
g_{\mu \nu} k^{\mu} k^{\nu}=0, d k^{\mu} / d \lambda+\Gamma_{\rho \sigma}^{\mu} k^{\rho} k^{\sigma}=0
$$

where $k^{\mu} \equiv d x^{\mu} / d \lambda$ and $\lambda$ is an affine parameter. Consider a light signal emitted at $\left(\vec{x}_{0}, t_{0}\right)$ in the initial direction described by a unit vector $\hat{n}$ satisfying $\hat{n} \cdot \hat{n}=1$ and let it have the form

$$
\vec{x}(t)=\vec{x}_{0}+\hat{n}\left(t-t_{0}\right)+\vec{x}_{p}(t)+\vec{x}_{p p}(t)
$$

where $\vec{x}_{p}(t)$ and $\vec{x}_{p p}(t)$ are the first and second post-Newtonian correction respectively. We obtain the solution needed for the second-order approximation by iterative methods.

Consider an observer (satellite, spacecraft) with the four-velocity $u^{\mu}$ who receives the signals from two different sources. The angle $\alpha$ between the directions of two incoming photons is given by the following expression:

$$
\cos \alpha=k_{I}^{\mu} P_{\mu}^{\rho} k_{I I}^{\sigma} P_{\rho \sigma}\left|k_{I}^{\mu} P_{\mu}^{\rho}\right|^{-1}\left|k_{I I}^{\sigma} P_{\rho \sigma}\right|^{-1}=f\left(g_{\mu \nu}, u^{\sigma}, k_{I}^{\rho}, k_{I I}^{\tau}\right),
$$

where $P_{\mu \nu}=g_{\mu \nu}+u_{\mu} u_{\nu}$ is a projection operator. Defining the angle $\delta \alpha$ to be the deflected angle from the original angle $\alpha_{0}$, and expanding $\cos \alpha$ around $\alpha_{0}$ to the second order, we have

$$
\delta \alpha_{p}=\cot \alpha_{0}-f \csc \alpha_{0}, \delta \alpha_{p p}=\cot \alpha_{0}-f \csc \alpha_{0}-\frac{1}{2}\left(\delta \alpha_{p}\right)^{2} \cot \alpha_{0}-\delta \alpha_{p}
$$

where $\delta \alpha_{p}$ and $\delta \alpha_{p p}$ are the deflection angles for the first and second post-Newtonian approximations. After a straightforward but lengthy calculation, we have

$$
\begin{gathered}
\delta \alpha=2(2+\bar{\gamma})(M / R)+(2+\bar{\gamma}) \bar{\gamma} \xi_{1}(M / 2 \pi R)-(2+\bar{\gamma})\left(M R / 2 r_{o s}^{2}\right) \\
+\left[\left(30+31 \bar{\gamma}+8 \bar{\gamma}^{2}\right) \pi-16(2+\bar{\gamma})^{2}\right]\left(M^{2} / 8 R^{2}\right)+2(2+\bar{\gamma}) J_{2}(M / R),
\end{gathered}
$$

for light passing the solar limb in the equatorial plane from outside the solar system. Here $M$ is the solar mass, $R$ is the solar radius and $J_{2}$ is the solar quadrupole moment parameter. The second term comes from the intermediate-range force and other terms agree with the former works.

The $2 \mathrm{PN}$ light trajectory obtained here is useful for obtaining $2 \mathrm{PN}$ range of deep space laser ranging missions ASTROD I and ASTROD (Ni 2004). A detailed paper on this topic will be presented in the future.

\section{Acknowledgement}

We thank the National Natural Science Foundation (Grant Nos 10475114 and 10778710) and the Foundation of Minor Planets of purple Mountain Observatory for support.

\section{References}

Epstein, R. \& Shapiro, I. I. 1980, Phys. Rev., D22, 2947; and references therein Richter, G. W. \& Matzner, R. A. 1982, Phys. Rev., D26, 1219; and references therein Xie, Y., et al. 2007, Adv. Sp. Res., doi: 10.1016/j.asr.2007.09.022, arXiv:0704.2991v2 Ni, W. T., et al. 2004, Class. Quantum Grav., 21, S641 